\title{
THE VLT-FLAMES TARANTULA SURVEY: THE FASTEST ROTATING O-TYPE STAR AND SHORTEST PERIOD LMC PULSAR-REMNANTS OF A SUPERNOVA DISRUPTED BINARY?
}

\author{
P. L. Dufton ${ }^{1}$, P. R. Dunstall ${ }^{1}$, C. J. Evans ${ }^{2}$, I. Brott ${ }^{3}$, M. Cantiello ${ }^{4,5}$, A. De Koter ${ }^{6,7}$, S. E. De Mink ${ }^{8,13}$, M. Fraser ${ }^{1}$, \\ V. Hénault-Brunet ${ }^{9}$, I. D. Howarth ${ }^{10}$, N. Langer ${ }^{4}$, D. J. Lennon ${ }^{11}$, N. Markova ${ }^{12}$, H. Sana ${ }^{6}$, AND W. D. TaYlor ${ }^{9}$ \\ ${ }^{1}$ Astrophysics Research Centre, School of Mathematics and Physics, Queen’s University Belfast, Belfast BT7 1NN, UK; p.dufton@qub.ac.uk \\ ${ }^{2}$ UK Astronomy Technology Centre, Royal Observatory Edinburgh, Blackford Hill, Edinburgh EH9 3HJ, UK \\ ${ }^{3}$ University of Vienna, Department of Astronomy, Türkenschanzstr. 17, A-1180 Vienna, Austria \\ ${ }^{4}$ Argelander Institut für Astronomie der Universität Bonn, Auf dem Hügel 71, 53121 Bonn, Germany \\ ${ }^{5}$ Kavli Institute for Theoretical Physics, Kohn Hall, University of California, Santa Barbara, CA 93106, USA \\ ${ }^{6}$ Astronomical Institute "Anton Pannekoek," University of Amsterdam, Postbus 94249, 1090 GE Amsterdam, The Netherlands \\ ${ }^{7}$ Astronomical Institute, Utrecht University, Princetonplein 5, 3584 CC Utrecht, The Netherlands \\ ${ }^{8}$ Space Telescope Science Institute, 3700 San Martin Drive, Baltimore, MD 21218, USA \\ ${ }^{9}$ Scottish Universities Physics Alliance, Institute for Astronomy, University of Edinburgh, Royal Observatory Edinburgh, Blackford Hill, Edinburgh EH9 3HJ, UK \\ ${ }^{10}$ Department of Physics and Astronomy, University College London, Gower Street, London WC1E 6BT, UK \\ ${ }^{11}$ ESA, Space Telescope Science Institute, 3700 San Martin Drive, Baltimore, MD 21218, USA \\ 12 Institute of Astronomy with NAO, Bulgarian Academy of Sciences, P.O. Box 136, 4700 Smoljan, Bulgaria \\ Received 2011 October 14; accepted 2011 October 31; published 2011 November 22
}

\begin{abstract}
We present a spectroscopic analysis of an extremely rapidly rotating late O-type star, VFTS102, observed during a spectroscopic survey of 30 Doradus. VFTS102 has a projected rotational velocity larger than $500 \mathrm{~km} \mathrm{~s}^{-1}$ and probably as large as $600 \mathrm{~km} \mathrm{~s}^{-1}$; as such it would appear to be the most rapidly rotating massive star currently identified. Its radial velocity differs by $40 \mathrm{~km} \mathrm{~s}^{-1}$ from the mean for 30 Doradus, suggesting that it is a runaway. VFTS102 lies 12 pc from the X-ray pulsar PSR J0537-6910 in the tail of its X-ray diffuse emission. We suggest that these objects originated from a binary system with the rotational and radial velocities of VFTS102 resulting from mass transfer from the progenitor of PSR J0537-691 and the supernova explosion, respectively.
\end{abstract}

Key words: Magellanic Clouds - pulsars: individual (PSR J0537-6910) - stars: early-type - stars: evolution stars: rotation

Online-only material: color figures

\section{INTRODUCTION}

In recent years, the importance of binarity in the evolution of massive stars has been increasingly recognized. This arises from most OB-type stars residing in multiple systems (Mason et al. 2009) and the significant changes to stellar properties that binarity can cause (see, for example, Podsiadlowski et al. 1992; Langer et al. 2008; Eldridge et al. 2011).

Here, we present a spectroscopic analysis of a rapidly rotating $\left(v_{\mathrm{eq}} \sin i \sim 600 \mathrm{~km} \mathrm{~s}^{-1}\right)$ O-type star in the 30 Doradus region of the Large Magellanic Cloud (LMC). Designated VFTS102 (Evans et al. 2011, hereafter Paper I) ${ }^{14}$ the star is rotating more rapidly than any observed in recent large surveys (Martayan et al. 2006; Hunter et al. 2009) and may also be a runaway. It lies less than 1 arcmin from the X-ray pulsar, PSR J0537-6910, which is moving away from it.

We suggest that VFTS102 might originally have been part of a binary system with the progenitor of the pulsar.

\section{OBSERVATIONS}

Spectroscopy of VFTS102 was obtained as part of the VLTFLAMES Tarantula Survey, covering the $3980-5050 \AA$ A region at a spectral resolving power of 7000 to 8500 . Spectroscopy of the $\mathrm{H} \alpha$ region was also available, although this was not used in the quantitative analysis. Details of the observations and initial data reduction are available in Paper I.

\footnotetext{
${ }^{13}$ Hubble Fellow.

14 Aliases include: ST92 1-32; 2MASS J05373924-6909510.
}

The spectra were normalized to selected continuum windows using a sigma-clipping rejection algorithm to exclude cosmic rays. No velocity shifts were observed between different epochs, although simulations (see Sana et al. 2009) indicate that 30\% of short period (less than 10 days) and effectively all longer term binaries would not have been detected. We have therefore assumed VFTS102 to be single and the sigma-clipped merged spectrum displays a signal-to-noise ratio of approximately 130 and 60 for the $4000-4500$ and $4500-5000 \AA$ regions, respectively.

An O9: Vnnne spectral classification was obtained by smoothing and rebinning the spectrum to an effective resolving power of 4000 and comparing with standards compiled for the Tarantula Survey (H. Sana et al. 2012, in preparation). The principle uncertainties arise from the extremely large rotational broadening and significant nebular contamination of the He I lines, with the two suffixes indicating extreme line broadening ("nnn") and an emission-line star ("e").

\section{ANALYSIS}

\subsection{Projected Rotational Velocity}

The large rotational broadening of the spectral features makes reliable measurements of the projected rotational velocity, $v_{\mathrm{eq}} \sin i$, difficult. We have used a Fourier transform (FT) approach as discussed by Simón-Díaz \& Herrero (2007), supplemented by fitting rotational broadened profiles $(\mathrm{PF})$ to the observed spectral features. 

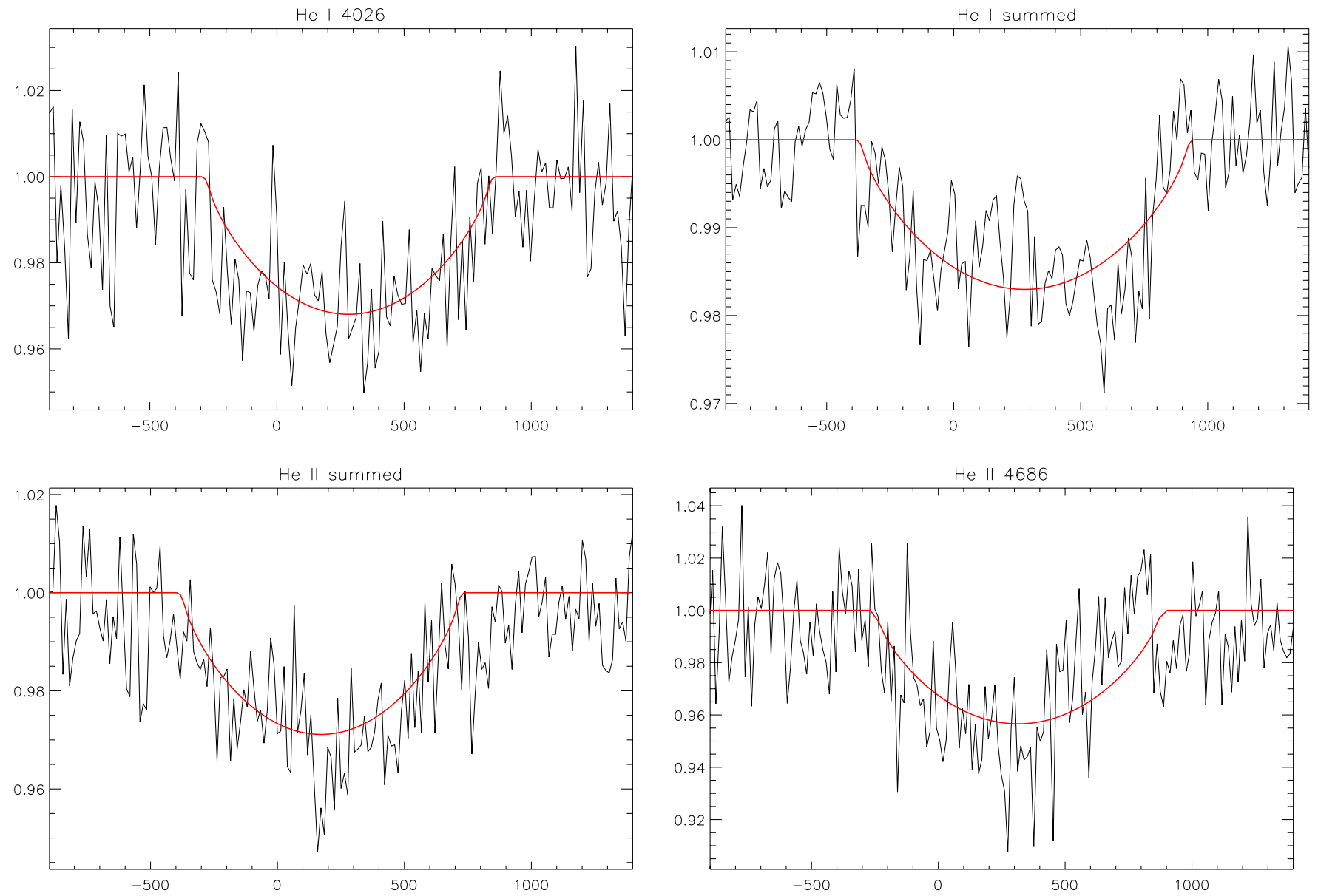

Figure 1. Observed spectra (in velocity space) for VFTS102 and rotationally broadened profiles for the He I line at $4026 \AA$, the combined profile for the He I lines at 4026, 4143, and $4387 \AA$, the combined profile for the He II lines at 4200 and $4541 \AA$, and the He II line at $4686 \AA$.

(A color version of this figure is available in the online journal.)

The Balmer lines have significant nebular emission and hence the weaker helium spectra were utilized, as illustrated in Figure 1. The He I line at $4471 \AA$, although well observed, also showed significant nebular emission and was not analyzed. By contrast the line at $4026 \AA$ showed no evidence of emission and yielded a plausible minimum in the FT for a $v_{\text {eq }} \sin i$ of $560 \mathrm{~km} \mathrm{~s}^{-1}$. The PF methodology leads to a slightly higher estimate $\left(580 \mathrm{~km} \mathrm{~s}^{-1}\right)$. The He I lines at 4143 and $4387 \AA$ were observed although they are relatively weak. These and the line at $4026 \AA$ were converted into velocity space, merged and analyzed. The two methodologies yielded effectively identical estimates of $640 \mathrm{~km} \mathrm{~s}^{-1}$; a similar procedure was undertaken for the He II lines at 4200 and 4541 A yielding $540 \mathrm{~km} \mathrm{~s}^{-1}$ (FT) and $510 \mathrm{~km} \mathrm{~s}^{-1}$ (PF). The He II line at $4686 \AA$ was found to be sensitive to the normalization with a $v_{\text {eq }} \sin i$ of $\sim 560 \mathrm{~km} \mathrm{~s}^{-1}$ being estimated.

The individual results should be treated with caution but overall they imply that this star is rotating near its critical velocity, with the mean value for the FT estimates being $580 \mathrm{~km} \mathrm{~s}^{-1}$. As discussed by Townsend et al. (2004), projected rotational velocities may be underestimated at these large velocities. For a B0 star rotating at $95 \%$ of the critical velocity, this underestimation will be approximately $10 \%$. Hence, our best estimate for the projected rotational velocity is $\sim 600 \mathrm{~km} \mathrm{~s}^{-1}$. A lower limit of $500 \mathrm{~km} \mathrm{~s}^{-1}$ has been adopted, while the upper value will be constrained by the critical velocity of approximately $700 \mathrm{~km} \mathrm{~s}^{-1}$ from the models of Brott et al. (2011).
This estimate is significantly higher than those $\left(\lesssim 370 \mathrm{~km} \mathrm{~s}^{-1}\right)$ found by Martayan et al. (2006) and Hunter et al. (2009) in their LMC B-type stellar samples. It is also larger than any of the preliminary estimates $\left(\lesssim 450 \mathrm{~km} \mathrm{~s}^{-1}\right)$ for $\sim 270$ B-type stars in the Tarantula survey, although other rapidly rotating O-type stars have been identified. As such it would appear to have the highest projected rotational velocity estimate of any massive star yet analyzed.

\subsection{Radial Velocity}

Radial velocities were measured by cross-correlating spectral features against a theoretical template spectrum taken from a grid calculated using the code TLUSTY (Hubeny 1988) - see Dufton et al. (2005) for details.

Five spectral regions were considered, viz. $\mathrm{H} \delta$ and $\mathrm{H} \gamma$ (with the cores excluded); He I at $4026 \AA ; 4630-4700 \AA$ with strong multiplets due to $\mathrm{C}_{\text {III }}$ and $\mathrm{O}$ II and an He II line; and 4000-4500 A (with nebular emission being excluded). The measurements are in excellent agreement with a mean value of $228 \pm 12 \mathrm{~km} \mathrm{~s}^{-1}$; if the error distribution is normally distributed the uncertainty in this mean value would be $6 \mathrm{~km} \mathrm{~s}^{-1}$.

From a study of $\sim 180$ presumably single O-type stars in the Tarantula survey, H. Sana et al. (2012, in preparation) find a mean velocity of $271 \mathrm{~km} \mathrm{~s}^{-1}$ with a standard deviation of $10 \mathrm{~km} \mathrm{~s}^{-1}$. Preliminary analysis of the B-type stars in the same survey has yielded $270 \pm 17 \mathrm{~km} \mathrm{~s}^{-1}$. VFTS102 lies more than 
Table 1

Properties of VFTS102

\begin{tabular}{lc}
\hline \hline Parameter & Estimate \\
\hline Spectral type & O9: Vnnne \\
$T_{\text {eff }}(\mathrm{K})$ & $36000 \pm 5000$ \\
$\log g\left(\mathrm{~cm} \mathrm{~s}^{-2}\right)$ & $3.6 \pm 0.5$ \\
$v_{\text {eq }} \sin i\left(\mathrm{~km} \mathrm{~s}^{-1}\right)$ & $600 \pm 100$ \\
$v_{\mathrm{r}}\left(\mathrm{km} \mathrm{s}^{-1}\right)$ & $228 \pm 6$ \\
$\log L / L_{\odot}$ & $5.0 \pm 0.2$ \\
\hline
\end{tabular}

two standard deviations away from these results, implying that it might be a runaway.

\subsection{Atmospheric Parameters}

While the equatorial regions of VFTS102 will have a lower gravity than the poles (because of centrifugal forces), and hence a lower temperature (because of von Zeipel gravity darkening), we first characterize the spectrum by comparison with those generated with spatially homogeneous models, convolved with a simple rotational-broadening function. We have used both our TLUSTY grid and FASTWIND calculations (Puls et al. 2005), adopting an LMC chemical composition. For the former, the strength of the He II spectrum implies an effective temperature ( $T_{\text {eff }}$ ) of $\sim 32,500-35,000 \mathrm{~K}$, while the wings of the Balmer lines lead to a surface-gravity estimate of $\sim 3.5 \mathrm{dex}(\mathrm{cgs})$. For the latter after allowing for wind effects, the corresponding parameters are $37,000 \mathrm{~K}$ and $3.7 \mathrm{dex}$. The helium spectra are consistent with a solar abundance but with the observational and theoretical uncertainties we cannot rule out an enhancement.

Given its projected equatorial rotation velocity, VFTS102 is almost certainly viewed at $\sin i \sim 1$. Hence the relatively cool, low-gravity equatorial regions will contribute significantly to the spectrum. Although their surface flux is lower than for the brighter poles, the analyses discussed above may underestimate the global effective temperature and gravity. However, the rotating-star models discussed below suggest that the effects are not very large. We therefore adopt global estimates for the effective temperature of $36,000 \mathrm{~K}$ and $3.6 \mathrm{dex}$ but note that the polar gravity could be as large as 4.0 dex. Varying the global parameters by the error estimates listed in Table 1 leads to significantly poorer matches between observation and the standard models, but, given the caveats discussed above, those errors should still be treated with caution.

For near critical rotational velocities, the stellar mass can be estimated. Howarth \& Smith (2001) show that the stellar mass can be written in terms of $\omega / \omega_{\mathrm{c}}{ }^{15} v_{e q}$, and the polar radius. Assuming that $\sin i \sim 1$ and adopting the critical velocities from our single star models, we can estimate the first two quantities. Additionally for any given value of $\omega / \omega_{\mathrm{c}}$, the polar radius can be inferred from the absolute visual magnitude and the unreddened $(B-V)$. The former can be estimated from the luminosity (see Section 3.4) and the latter from our effective temperature estimate and the LMC broadband intensities calculated by Howarth (2011). We find $M \gtrsim 20 M_{\odot}$ for $v_{\text {eq }} \sim 600 \mathrm{~km} \mathrm{~s}^{-1}$ and $T_{\text {eff }} \lesssim 38,000 \mathrm{~K}$. Only by adopting a smaller value for $v_{e q}$ can we push the mass limit down, but even with $v_{\mathrm{eq}} \sim 500 \mathrm{~km} \mathrm{~s}^{-1}$ the mass must exceed $\sim 17 M_{\odot}$.

\footnotetext{
15 The ratio of the equatorial angular velocity to that at which the centrifugal
} acceleration equals the gravitational acceleration.

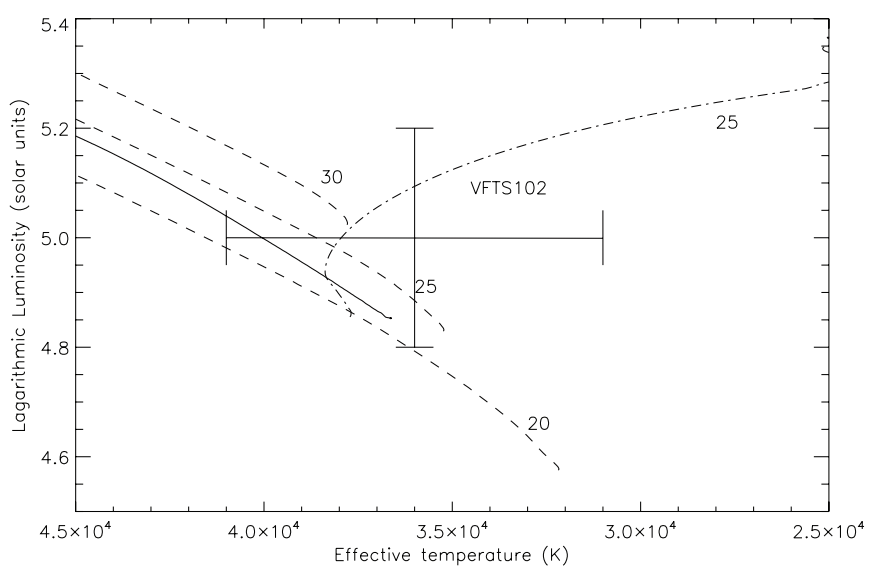

Figure 2. H-R diagram showing the estimated position of VFTS102. The evolutionary tracks (identified by their mass) have rotational velocities of approximately $600 \mathrm{~km} \mathrm{~s}^{-1}$ (dashed lines) and $400 \mathrm{~km} \mathrm{~s}^{-1}$ (dashed-dot line). Also shown is the evolution of the secondary star following mass transfer for the binary model of Cantiello et al. (2007; solid line).

\subsection{Luminosity}

From extant photometry (see Paper I), the $(B-V)$ color of VFTS102 is 0.35 , implying an $E(B-V)$ of 0.6 using colors calculated from our TLUSTY grid. Adopting a standard reddening law leads to a logarithmic luminosity (in solar units) of 5.0 dex, with an $E(B-V)$ error of \pm 0.1 corresponding to an uncertainty of \pm 0.1 dex. However, there are other possible sources of error, for example, deviations from a standard reddening law, and hence we have adopted a larger random error estimate of \pm 0.2 dex.

As VFTS102 is an Oe-type star, its intrinsic colors may be redder than predicted by our TLUSTY grid and indeed an infrared excess is found from published (de-reddened) Two Micron All Sky Survey (2MASS) photometry. Inspection of a $K$-band VISTA image shows no evidence of contamination by nearby sources. Further evidence for circumstellar material is found in the strong $\mathrm{H} \alpha$ emission, which is double peaked as is the nearby $\mathrm{He}$ I line at $6678 \AA$, which supports our adoption of a $\sin i \sim 1$. Additionally, there are weak doublepeaked Fe II emission features (e.g., at $4233 \AA$ ), consistent with an Oe-type classification. Unfortunately, our photometry and spectroscopy are not contemporaneous but if VFTS102 was in a high state when the optical photometry was taken, we may have overestimated the luminosity of the central star (see de Wit et al. 2006 for color and magnitude variations of Be stars).

\section{PAST AND FUTURE EVOLUTION}

Stellar evolution calculations for both single and binary stars are available in the literature (see Maeder \& Meynet 2011). For very fast rotation, they suggest that rotational mixing is so efficient that stars may evolve quasi-chemically homogeneously (Maeder 1987; Woosley \& Heger 2006; Cantiello et al. 2007; de Mink et al. 2009; Brott et al. 2011). However, with different physical assumptions, models do not evolve chemically homogeneously even for the fastest rotation rates (Cantiello et al. 2007; Ekström et al. 2008).

\subsection{Single Star Evolution}

Figure 2 illustrates evolutionary tracks for LMC single stars calculated using the methodology of Brott et al. (2011) for an initial equatorial rotational velocity of $600 \mathrm{~km} \mathrm{~s}^{-1}$, together with 
that for a more slowly rotating model. The former are evolving chemically homogeneously while the latter follows a "normal" evolutionary path. Ekström et al. (2008) calculated models for a range of metallicities and masses between 3 and $60 M_{\odot}$ but found that the stars followed normal evolutionary paths even for near critical rotational velocities.

The estimated parameters of VFTS102 are consistent with our tracks for initial masses of $\sim 20-30 M_{\odot}$. Our models show a relatively rapid increase in the surface helium abundance due to their homogeneous evolution. For example, the $25 M_{\odot}$ model shows an enrichment of a factor of 2 after approximately 4 million years and when the effective temperature has increased to approximately $39,000 \mathrm{~K}$. By contrast the models of Ekström et al. (2008) show no significant helium abundance implying that an accurate helium abundance estimate for VFTS102 would help constrain the physical assumptions.

\subsection{Binary Star Evolution}

Below, we first discuss the environment of VFTS102 and then consider a possible evolutionary scenario.

\subsubsection{A Pulsar Near VFTS102}

VFTS102 lies in a complex environment near the open cluster NGC 2060. In particular it lies close to a young X-ray pulsar PSR J0537-6910 (Marshall et al. 1998) and the Crab-like supernova remnant (SNR) B0538-691 (Micelotta et al. 2009). VFTS102 has an angular separation of approximately 0.8 arcmin from PSR J0537-6910 implying a spatial separation (in the plane of the sky) of approximately $12 \mathrm{pc}$.

The X-ray emission consists of a pulsed localized component and a more spatially diffuse component, with the latter providing the majority of the energy. The diffuse component was identified in ROSAT and ASCA observations by Wang \& Gotthelf (1998a) and interpreted as coming from ram-pressure-confined material with the X-ray pulsar being identified soon afterward by Marshall et al. (1998). Wang \& Gotthelf (1998b) analyzed ROSAT HRI observations and suggested that the emission could come from the remnants of a bow shock if the pulsar was moving with a velocity of $\sim 1000 \mathrm{~km} \mathrm{~s}^{-1}$. Wang et al. (2001) subsequently analyzed higher spatial resolution Chandra observations, which clearly delineated this emission and implied that the pulsar was moving away from VFTS102. See, however, Chen et al. (2006) for an alternative explanation of the X-ray morphology, which does not imply a high velocity for the pulsar. Figure 3 superimposes these emission contours onto a Hubble Space Telescope (HST) optical image with VFTS102 being near the tail of these contours. As discussed by Wang et al. (2001) the spatial distribution of the diffuse X-ray emission and the SNR optical emission are well correlated. Differences probably arise from a foreground dark cloud and photoionization and mechanical energy input from the nearby open cluster.

Timing measurements imply that the pulsar has a characteristic age of 5000 years (Marshall et al. 1998), consistent with the age estimate of Wang \& Gotthelf (1998b) from analysis of X-ray emission. Spyrou \& Stergioulas (2002) discuss the estimation of ages from spin rates and find the results to be sensitive to both the breaking index and the composition of the pulsar core. Indeed, phase-connected braking index measurements for young pulsars (see Zhang et al. 2001 and references therein) yield breaking indices lower than the $n=3$ normally adopted with corresponding increases in the characteristic ages. Additionally, Chu et al. (1992) found an age of approximately 24,000 years from the kinematics of the SNR.

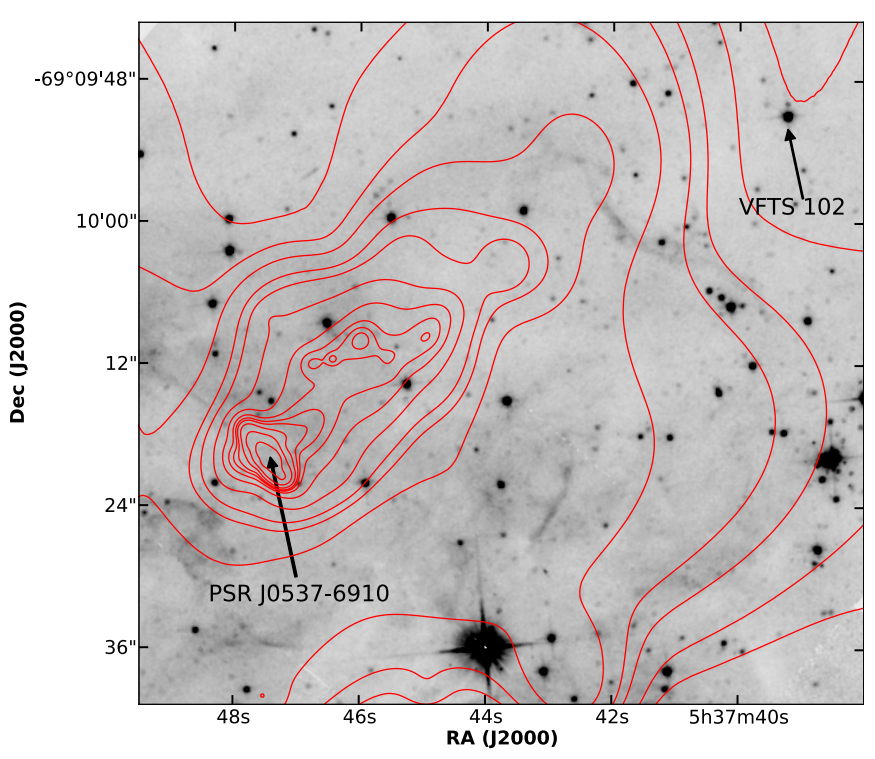

Figure 3. HST WFPC2 $V$-band (F606W filter) image with contours from the smoothed Chandra HRC-I image overlaid. The positions of VFTS102 and PSR J0537-6910 are labeled.

(A color version of this figure is available in the online journal.)

Adopting an age of 5000 years would imply that if these objects had been part of a binary system, their relative velocity $\left(v_{\mathrm{s}}\right)$ in the plane of the sky would be approximately $2500 \mathrm{~km} \mathrm{~s}^{-1}$. Increasing this age to 24,000 years would then imply $v_{\mathrm{s}} \sim$ $500 \mathrm{~km} \mathrm{~s}^{-1}$. These values, although large, are consistent with a pulsar velocity of $1000 \mathrm{~km} \mathrm{~s}^{-1}$ in the model of Wang \& Gotthelf (1998b) and of $\sim 600 \mathrm{~km} \mathrm{~s}^{-1}$ from the separation of the diffuse X-ray and radio emission (Wang et al. 2001). Additionally, Hobbs et al. (2005) found a mean space velocity of approximately $400 \mathrm{~km} \mathrm{~s}^{-1}$ for a sample of young pulsars with velocities as high as $1600 \mathrm{~km} \mathrm{~s}^{-1}$. From the theoretical point of view, Stone (1982) found supernova kick velocities normally in excess of $300 \mathrm{~km} \mathrm{~s}^{-1}$, while more recently Eldridge et al. (2011) estimated kick velocities for a single neutron star of more than $1000 \mathrm{~km} \mathrm{~s}^{-1}$ with a mean value of $\sim 500 \mathrm{~km} \mathrm{~s}^{-1}$.

\subsubsection{A Binary Evolution Scenario for VFTS102}

While the fast rotation of VFTS102 might be the result of the star formation process, it could also have arisen from spinup due to mass transfer in a binary system (Packet 1981). A subsequent supernova explosion of the donor star could then lead to an anomalous radial velocity for VFTS102 (Blaauw 1961; Stone 1982). The nearby pulsar and SNR make this an attractive scenario. Of course, we cannot eliminate other possible scenarios, e.g., dynamical ejection from a cluster (see Gvaramadze \& Gualandris 2011) but it is unclear whether these could produce the very large rotational velocity of VFTS102.

Cantiello et al. (2007) have modeled a binary system with initial masses of 15 and $16 M_{\odot}$ adopting Small Magellanic Cloud metallicity. After mass transfer the primary exploded as a Type $\mathrm{Ib} / \mathrm{c}$ supernova. At that stage the secondary has a mass of approximately $21 M_{\odot}$, a rotational velocity close to critical, and a logarithmic luminosity of approximately $4.9 \mathrm{dex}$ (see Figure 2 for its subsequent evolution). These properties closely match the estimates for VFTS102 summarized in Table 1.

Based on grids of detailed binary evolutionary models (Wellstein et al. 2001; de Mink et al. 2007), the initial masses of the two components of such a binary system should be 
comparable, with $M_{2} / M_{1} \gtrsim 0.7$. If the initial mass of the secondary was in the range of $14-18 M_{\odot}$, that of the primary would need to be smaller than about $25 M_{\odot}$. This agrees with the estimated initial mass of the supernova progenitor based on the kinematics of the SNR (Micelotta et al. 2009).

In this scenario, it takes the primary star about $11 \mathrm{Myr}$ to evolve to the supernova stage. While the most massive stars in 30 Doradus have ages of a few million years (Walborn et al. 1999), there is also evidence for different massive stellar populations with ages ranging up to about $10 \mathrm{Myr}$ (Walborn \& Blades 1997). Recently, De Marchi et al. (2011) have undertaken an extensive study of lower mass $\left(\lesssim 4 M_{\odot}\right)$ main sequence and pre-main-sequence stars in 30 Doradus. They obtain a median age of 12 Myr with ages of up 30 Myr. Hence, it would appear possible that the putative binary system formed in the vicinity of 30 Doradus approximately $10 \mathrm{Myr}$ ago and underwent an evolutionary history similar to that modeled by Cantiello et al. (2007).

While the evolutionary link between VFTS102 and the pulsar is an attractive scenario, the offset between VFTS102 and the apparent center of the radio emission (see Wang et al. 2001) remains to be explained. Proper motion information would be extremely valuable to further test this hypothesis. PSR J0537-6910 has not been definitely identified in other wavelength regions. Mignani et al. (2005) using Advanced Camera for Surveys imaging from the HST found two plausible identifications that would imply an optical luminosity similar to the Crab-like pulsars. A radio survey by Manchester et al. (2006) only yielded an upper limit to its luminosity consistent with other millisecond pulsars. However, estimates for both components may be obtained from the HST proper motion study (Program: 12499; PI: D. J. Lennon) that is currently underway.

\subsection{Evolutionary Future}

Irrespective of the origin of VFTS102, it is interesting to consider its likely fate. Stellar evolutionary models of rapidly rotating stars have recently been generated by Woosley \& Heger (2006) and Yoon et al. (2006). The latter consider the fate of objects with rotational velocities up to the critical value $\left(v_{\mathrm{c}}\right)$. The evolution is shown to depend not only on initial mass and rotational velocity but also on the metallicity. In particular, gamma-ray bursts (GRBs) are predicted to occur only at subsolar metallicities.

Based on our single star models, VFTS102 has a rotational velocity above $\sim 0.8 v_{\mathrm{c}}$ and is thus expected to evolve quasichemically homogeneously. While Yoon et al. (2006) and Woosley \& Heger (2006) estimate the metallicity threshold for GRB formation from chemically homogeneous evolution to be somewhat below the LMC metallicity, the latter note its sensitivity to the mass-loss rate (Vink \& de Koter 2005). Indeed, all our most rapidly rotating $20-30 M_{\odot}$ models are evolving chemically homogeneously throughout core hydrogen burning (Figure 2), a prerequisite to qualify for a GRB progenitor. In any case, within the context of homogeneous evolution, VFTS102 is expected to form a rapidly rotating black hole and a Type Ic hypernova. This conjecture remains the same within the binary scenario of Cantiello et al. (2007).

Assuming a space velocity of $40 \mathrm{~km} \mathrm{~s}^{-1}$ for VFTS102 (compatible with its anomalous radial velocity), our evolutionary models imply that VFTS102 will travel $\sim 300-400$ pc before ending its life. This is consistent with the finding of Hammer et al. (2006) that the locations of three nearby GRBs were found several hundred parsecs away from their most likely progenitor birth locations (see, however, Margutti et al. 2007; Wiersema et al. 2007; Han et al. 2010).

\section{CONCLUSIONS}

VFTS102 has a projected rotational velocity far higher than those found in previous surveys of massive stars in the LMC, and indeed it would appear to qualify as the most rapidly rotating massive star yet identified. With a luminosity of $10^{5} L_{\odot}$ we estimate its current mass to be approximately $25 M_{\odot}$. Its extreme rotation, peculiar radial velocity, and proximity to the X-ray pulsar PSR J0537-6910 and to an SNR suggest that the star is the result of binary interaction.

It is proposed that VFTS102 and the pulsar originated in a binary system with mass transfer spinning-up VFTS102 and the supernova explosion imparting radial velocity kicks to both components. If evolving chemically homogeneously, as suggested by recent models, VFTS 102 could become a GRB or hypernova at the end of its life. Additionally, it may provide a critical test case for chemically homogeneous evolution.

S.d.M. acknowledges the NASA Hubble Fellowship Grant HST-HF-51270.01-A awarded by STScI, operated by AURA for NASA, Contract NAS 5-26555. N.M. acknowledges support from the Bulgarian NSF (DO 02-85). We thank Eveline Helder, Paul Quinn, Stephen Smartt, Jorick Vink, and Nolan Walborn for useful discussions. This paper makes use of spectra obtained as part of the VLT-FLAMES Tarantula Survey (ESO programme 182.D-0222).

\section{Facilities: VLT:Kueyen (FLAMES)}

\section{REFERENCES}

Blaauw, A. 1961, Bull. Astron. Inst. Netherlands, 15, 265

Brott, I., de Mink, S. E., Cantiello, M., et al. 2011, A\&A, 530, A115

Cantiello, M., Yoon, S.-C., Langer, N., \& Livio, M. 2007, A\&A, 465, L29

Chen, Y., Wang, Q.-D., Gotthelf, E.-V., et al. 2006, ApJ, 651, 237

Chu, Y.-H., Kennicutt, R. C., Jr., Schommer, R. A., \& Laff, J. 1992, AJ, 103, 1545

de Mink, S. E., Cantiello, M., Langer, N., et al. 2009, A\&A, 497, 243

de Mink, S. E., Pols, O. R., \& Hilditch, R. W. 2007, A\&A, 467, 1181

De Marchi, G., Paresce, F., Panagia, N., et al. 2011, ApJ, 730, 27

de Wit, W. J., Lamers, H. J. G. L. M., Marquette, J. B., \& Beaulieu, J. P. 2006, A\&A, 456, 1027

Dufton, P. L., Ryans, R. S. I., Trundle, C., et al. 2005, A\&A, 434, 1125

Ekström, S., Meynet, G., Maeder, A., et al. 2008, A\&A, 478, 467

Eldridge, J. J., Langer, N., \& Tout, C. A. 2011, MNRAS, 414, 3501

Evans, C. J., Taylor, W. D., Hénault-Brunet, V., et al. 2011, A\&A, 530, A108

Gvaramadze, V. V., \& Gualandris, A. 2011, MNRAS, 410, 304

Hammer, F., Flores, H., Schaerer, D., et al. 2006, A\&A, 454, 103

Han, X. H., Hammer, F., Liang, Y. C., et al. 2010, A\&A, 514, A24

Hobbs, G., Lorimer, D. R., Lyne, A. G., \& Kramer, M. 2005, MNRAS, 360, 974

Howarth, I. D. 2011, MNRAS, 413, 1515

Howarth, I. D., \& Smith, K. C. 2001, MNRAS, 327, 353

Hubeny, I. 1988, Comput. Phys. Commun., 52, 103

Hunter, I., Brott, I., Langer, N., et al. 2009, A\&A, 496, 841

Langer, N., Cantiello, M., Yoon, S.-C., et al. 2008, in IAU Symp., Massive Stars as Cosmic Engines, ed. F. Bresolin, P. A. Crowther, \& J. Puls (Cambridge: Cambridge University press, 2008), 250, 167

Maeder, A. 1987, A\&A, 178, 159

Maeder, A., \& Meynet, G. 2011, Rev. Mod. Phys. (arXiv:1109.6171)

Manchester, R. N., Fan, G., Lyne, A. G., Kaspi, V. M., \& Crawford, F. 2006, ApJ, 649, 235

Margutti, R., Chincarini, G., Covino, S., et al. 2007, A\&A, 474, 815

Marshall, F. E., Gotthelf, E. V., Zhang, W., Middleditch, J., \& Wang, Q. D. 1998, ApJ, 499, L179

Martayan, C., Frémat, Y., Hubert, A.-M., et al. 2006, A\&A, 452, 273

Mason, B. D., Hartkopf, W. I., Gies, D. R., Henry, T. J., \& Helsel, J. W. 2009, AJ, 137,3358 
Micelotta, E. R., Brandl, B. R., \& Israel, F. P. 2009, A\&A, 500, 807

Mignani, R. P., Pulone, L., Iannicola, G., et al. 2005, A\&A, 431, 659

Packet, W. 1981, A\&A, 102, 17

Podsiadlowski, P., Joss, P. C., \& Hsu, J. J. L. 1992, ApJ, 391, 246

Puls, J., Urbaneja, M. A., Venero, R., et al. 2005, A\&A, 435, 669

Sana, H., Gosset, E., \& Evans, C. J. 2009, MNRAS, 400, 1479

Simón-Díaz, S., \& Herrero, A. 2007, A\&A, 468, 1063

Spyrou, N. K., \& Stergioulas, N. 2002, A\&A, 395, 151

Stone, R. C. 1982, AJ, 87, 90

Townsend, R. H. D., Owocki, S. P., \& Howarth, I. D. 2004, MNRAS, 350, 189

Vink, J. S., \& de Koter, A. 2005, A\&A, 442, 587
Walborn, N. R., Barbá, R. H., Brandner, W., et al. 1999, AJ, 117, 225

Walborn, N. R., \& Blades, J. C. 1997, ApJS, 112, 457

Wang, Q. D., \& Gotthelf, E. V. 1998, ApJ, 494, 623

Wang, Q. D., \& Gotthelf, E. V. 1998, ApJ, 509, L109

Wang, Q. D., Gotthelf, E. V., Chu, Y.-H., \& Dickel, J. R. 2001, ApJ, 559, 275

Wellstein, S., Langer, N., \& Braun, H. 2001, A\&A, 369, 939

Wiersema, K., Savaglio, S., \& Vreeswijk, P. M. 2007, A\&A, 464, 529

Woosley, S. E., \& Heger, A. 2006, ApJ, 637, 914

Yoon, S.-C., Langer, N., \& Norman, C. 2006, A\&A, 460, 199

Zhang, W., Marshall, F. E., Gotthelf, E. V., Middleditch, J., \& Wang, Q. D. 2001, ApJ, 554, L177 